\title{
Bilateral Symmetry Based Approach for Joint Detection of Landmarks in Retinal Images
}

\author{
Ayush Tewari \\ CVIT \\ IIIT Hyderabad \\ Hyderabad, India \\ Email: ayush.tewari@students.iiit.ac.in
}

\author{
Dhwanit Gupta \\ CVIT \\ IIIT Hyderabad \\ Hyderabad, India \\ Email: dhwanit.gupta@students.iiit.ac.in
}

\author{
Jayanthi Sivaswamy \\ CVIT \\ IIIT Hyderabad \\ Hyderabad, India \\ Email: jsivaswamy@iiit.ac.in
}

\begin{abstract}
Optic disk (OD) and macula are anatomical structures in retinal images. Their detection is of interest in computer assisted diagnostic (CAD) algorithm development. We propose a joint detection algorithm which exploits the bilateral symmetry present in retinal images and present a method to detect OD and macula. A vessel density based metric is proposed to determine the axis of symmetry which is used along with appearance features and geometric constraint on the OD and macula for their detection. The proposed method has been evaluated on a large number of images drawn from 4 public and one private dataset. Obtained results show that our method has consistently high detection rate across the datasets. Since the detection is also very fast, the proposed method offers a good solution for CAD based disease screening.
\end{abstract}

\section{INTRODUCTION}

Localization of anatomical landmarks is an essential task in automated medical image analysis. The optic disc (OD) and macula are important anatomical landmarks in retinal images. Their positions are useful in defining a retinal coordinate system for analyzing the distribution of abnormalities in a retinal image. The ETDRS protocol used in acquiring images for diabetic retinopathy uses a co-ordinate system centered at the macula to analyze the distribution of lesions in Diabetic Retinopathy. The Artery-Vein width ratio, which is an important indicator of various systemic diseases like hypertensive retinopathy is measured in a specific region defined relative to the OD center. Computer assisted diagnosis (CAD) algorithms which aim to detect lesions also localize and mask the OD and macula as a pre-processing step to remove false positives during lesion detection. In the present

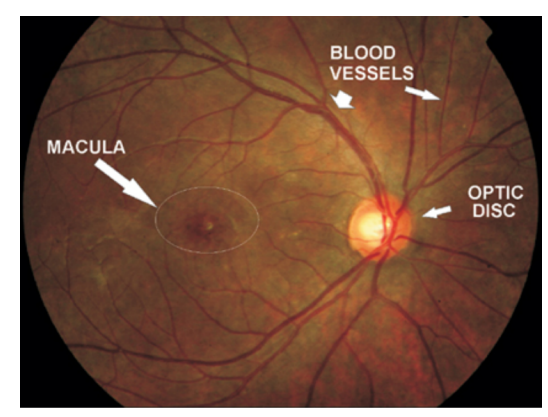

Fig. 1. A labelled CFI image work, we propose a solution for the joint localization of OD and macula in a color fundus image(CFI). This is based on exploiting the bilateral symmetry of the retinal vasculature at a global level as well as local low-level appearance features of OD and macula. The rest of the paper is structured as follows : section II briefly examines some previous works on this problem. Section III provides with the details of the proposed method. This is followed by results and their discussion in section IV.

\section{RELATED WORK}

OD and Macula detection have received significant attention from the research community. These efforts follow one of two approaches: separate strategies for detection of OD and macula or a single strategy for the joint detection of OD and macula.

Several techniques for OD detection have been proposed. These include clustering [11] and projections along multiple directions operators [13]. Other methods based on Hough transform [18], regression [15], template matching [10] [3], matched filtering [2] and mathematical morphology [20] have also been attempted. These methods vary in their robustness to the presence of pathologies, uneven illumination and images with low contrast between the OD and the retinal background.

Model based approaches try to overcome some of these difficulties by formulating OD detection as an optimization problem. They rely on the properties of the vessel network to localize the OD such as a region of convergence of thick vessels. Hence, a parabola, whose vertex is the OD location, is fit to the major vessel arcade [14],[5]. These methods are more robust but require accurate vessel extraction and detection of the main vessel arcade.

Macula detection has received relatively less attention. Macula detection has received relatively less attention. A common assumption in all methods is the knowledge of OD location [19] [4] [6]. This knowledge is to augment the vessel arcade location [4] or used along with the information on size of the OD to roughly locate the macula [19]. Another approach is to use regression to determine the location of the macula [15].

Joint detection of OD and macula has also been attempted. An ensemble based technique is proposed in [17] to combine the results of different, existing methods for OD and macula detection and find probable regions (hotspots). Geometric 
constraints are used to obtain the final location. The circular symmetry of the OD and macula is exploited in [7] to find bright and dark regions possessing circular symmetry and the false positive locations are rejected using the knowledge that OD and macula are regions with high and low vessel densities, respectively. The desired candidate regions are found using a fast radial symmetry transform (RST) proposed in [12]. In order to suppress the vessel structures which affect the symmetry computation, a rough vessel detection and inpainting are done prior to the the RST computation. Inpainting is generally a computationally expensive process.

In a retinal image, with macula at the centre, the vessel tree exhibits a bilateral symmetry about an axis which passes through the OD and macula as seen in Fig. III. Furthermore, the distance between the macula and OD is always $5 d$ where $d$ is the diameter of the OD. In this paper, we propose a fast method to jointly detect OD and macula based on this bilateral symmetry and the geometric constraint that the distance between the centre of the macula and OD is fixed. We propose using a vessel density based metric for the determining the bilateral symmetry. This obviates the need for accurate vessel extraction and aids fast detection of OD and macula. Thus, our approach relies on the distribution of the pixels of the vessels over a region rather than their geometry.

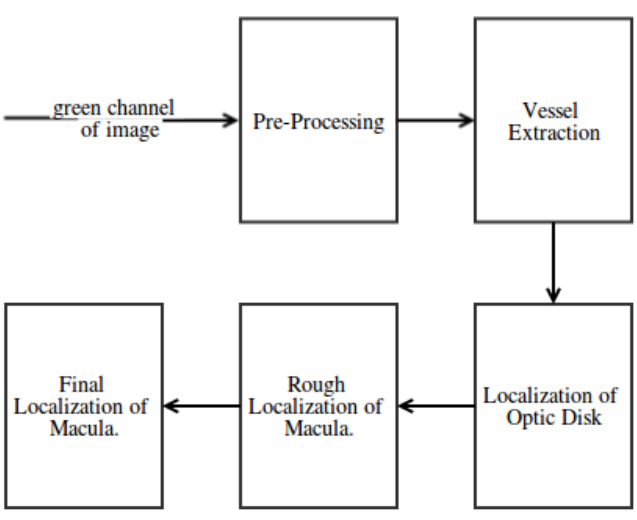

Fig. 2. Processing steps in the proposed algorithm

\section{METHOD}

Macular-centric view is a standard protocol used in diabetic retinopathy. In this view, with the macula at the centre, the OD can appear to the left or right depending on the eye being imaged. In both cases, and the image is roughly bilaterally symmetric with the axis of symmetry passing through the macula and OD (see figure 3). Thus, the axis of bilateral symmetry is a line that divides the retinal image into two regions of approximately equal vessel density.

The computation of the line of symmetry can be formulated as an unconstrained discrete optimization problem. Let us define a line using the slope-intercept form, $y=m x+c$ with $m$ denoting its slope and $c$ denoting its intercept on the y-axis.

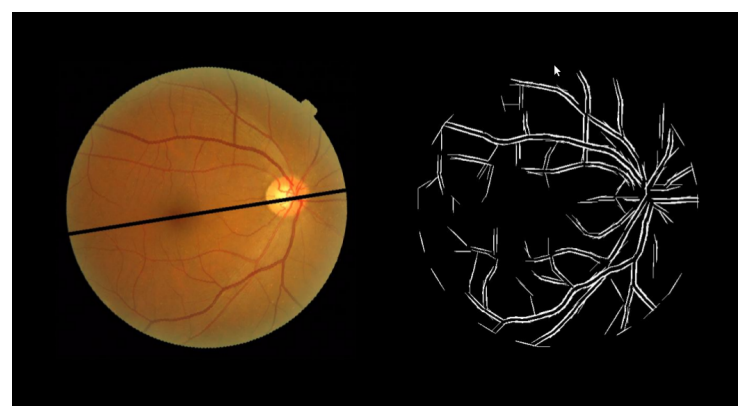

Fig. 3. Sample retinal image (left) and its rough vessel map (right). The axis of bilateral symmetry is shown as a black line in the colour image.

Each line in a plane divides the plane into two regions $(R)$. denoted as $R_{i}$ with $i \in\{$ upper, lower $\}$. We define the upper $R_{\text {upper }}$ and lower $R_{\text {lower }}$ regions as

$$
\begin{aligned}
& R_{\text {upper }}=\{(x, y) \mid y-m x-c>0\} \\
& R_{\text {lower }}=\{(x, y) \mid y-m x-c<0\}
\end{aligned}
$$

We also define the density $(D)$ of a region as follows

$$
D(R)=\frac{\text { Number of vessel pixels in } R}{\text { Area of } R}
$$

The axis of bilateral symmetry is defined as the line $L$ that minimizes the difference in the vessel density in regions above and below the line. Using the previously defined notations, this can be written mathematically as ,

$$
L_{\text {symm }}(m, c)=\underset{m, c}{\arg \min }\left\{\left|D\left(R_{\text {upper }}\right)-D\left(R_{\text {lower }}\right)\right|\right\} .
$$

Optimization of the above objective function is challenging as it has a number of local minima and an exhaustive search for all possible local minima is computationally expensive. It should also be noted, that a number of abnormalities may be present in a given retinal image, all of which act as noise sources and can impede the solution. Hence, directly that solving equation 4 is not advisable. Instead, we find a rough solution for the line and detect the OD first. Next, we use the OD location and the geometric constraint that the macula should be at a distance $5 r$ from the OD centre to further constrains the optimisation problem. These constraints make the optimization problem significantly tractable by reducing the number of local minima.

The processing steps are outlined in Figure. 2. We shall now present the details of each individual module, namely, the preprocessing of the retinal image, vessel detection followed by the OD and macula localization.

\section{A. Pre-processing}

All processing is done on the green channel of the given image as it is known to have the maximum contrast, which is an important factor for reliable OD and macula detection. For a specific dataset, we use the information of average OD diameter obtained from the dataset itself. In order to handle 
varying image sizes (due to camera type and settings) an input image is first resized to a standard size and then smoothed using a Gaussian kernel $(\mu=0, \sigma=51)$.

\section{B. Detection of Vessels}

Equation 3 depends only on vessel statistics. Hence, a basic vessel detection method is adequate. This flexibility on the part of the proposed approach helps achieve a fast detection which is useful in large-scale screening scenarios where speed is of the essence.

In our experiments, we used a simple and robust vessel segmentation algorithm [21] based on morphological operations. This method has been shown to have less false positives which is required for our algorithm to give correct output.

\section{Detection of Optic Disk}

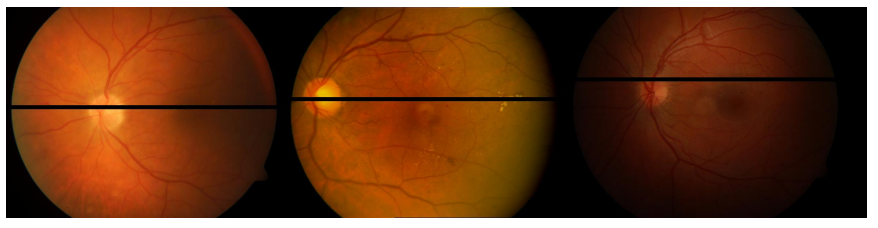

Fig. 4. Result of finding horizontal lines (in black) of bilateral symmetry in 3 sample images.

The OD is a bright, roughly circular structure and it forms the base of the vessel tree. Based on these observations, we define a pixel $p$ to be desired OD location if it satisfies the following conditions:

1) The difference between the average brightness in its immediate and distant (circular) neighborhoods is large. The distant neighborhood captures the context of OD in the image. This condition ensures that the region is locally bright.

2) The vessel density within a neighborhood is large. This condition helps in distinguishing the OD from other bright lesions in the image.

3) The distance between the pixel $p$ and the axis of symmetry is small.

The axis of symmetry is assumed to be horizontal for minimising the computations. This is simply found by finding the row about which the vessel density is roughly equal. This is a reasonable assumption as it always holds in a standard macula centric view, in the absence of any rotation. Formally,

$$
L_{h}(0, c)=\underset{c}{\arg \min }\left\{\left|D\left(R_{\text {upper }}\right)-D\left(R_{\text {lower }}\right)\right|\right\} .
$$

The third condition is particularly effective in cases where the illumination is considerably low and it is difficult to identify the location of the OD based on just the first two conditions. Fig 4 shows this line for some images.

The OD location is found using the above conditions as follows. At each pixel location $(\mathrm{i}, \mathrm{j})$ a 4-dimensional vector is defined: $F_{i j}=\left\{S_{r}^{i j}, S_{1.5 r}^{i j}, V_{r}^{i j}, d_{L(0, c)}^{i j}\right\}$, where $L(0, c)$ is the horizontal line of symmetry and $r$ is the radius of the OD. The elements of the vector are as defined in table I.
TABLE I

DEFINITION OF VARIOUS FEATURES USED

\begin{tabular}{|c|c|}
\hline Terms & Explanation \\
\hline$S_{r}^{i j}$ & $\begin{array}{c}\text { Sum of pixel intensities in a circular } \\
\text { neighborhood of radius r around the }(i, j)^{t h} \text { pixel. }\end{array}$ \\
\hline$V_{r}^{i j}$ & $\begin{array}{c}\text { Density of vessels, } D_{R_{i j}} \text { where } R_{i j} \text { is a circular } \\
\text { neighborhood of radius } \mathrm{r} \text { around the }(i, j)^{t h} \text { pixel. }\end{array}$ \\
\hline$d_{L(0, c)}^{i j}$ & Perpendicular distance of $(i, j)^{t h}$ pixel from $L(0, c)$. \\
\hline$\rho_{L(m, c)}^{i j}$ & Length of normal from $(i, j)$ and $L(m, c)$. \\
\hline
\end{tabular}

We train a linear SVM classifier on the above 4-dimensional feature set to get a probability score for each pixel being the OD centre. The probability map is smoothed by a Gaussian filter before selecting the pixel with maximum probability as the OD centre.

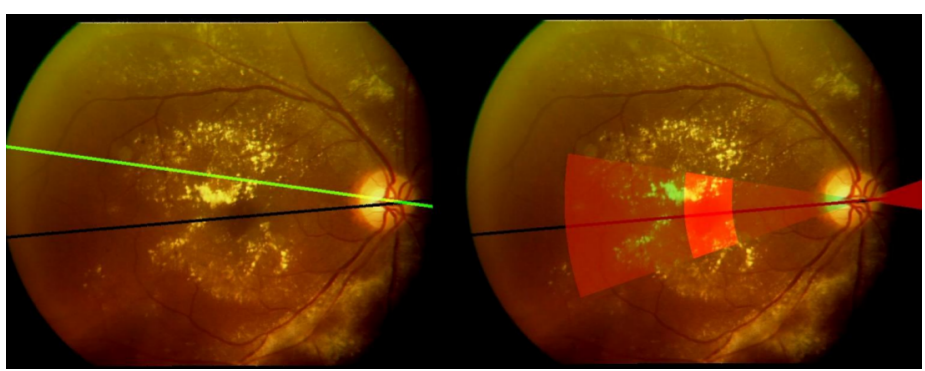

Fig. 5. Region of interest for macula (ROI). Left Candidate lines of symmetry. Right The sector with center at OD and the ROI, shown as the shaded regions.

\section{Detection of Macula}

Our localization of the macula is based on two premises - 1) the line of bilateral symmetry must pass through the macula and 2) macula is a roughly circular, dark structure, albeit without a sharply defined boundary as in the case of the OD.

1) Rough Localization: The equation 4 with the constraint that the line has to pass through the centre of OD (whose position is know now) is solved to find the candidate axes of symmetry. The results will be a set of lines radiating from the OD centre. An example with two lines is shown in the left image in Fig. 5.

The desired macula location is a point on one of the lines lying roughly $5 r$ ( $r$ being the radius of OD) from the OD centre. The latter helps reduce the search space for the macula. A sector with its apex at OD centre and of width $2 \mathrm{deg}$ is defined about every candidate line. Imposing the geometric constraint that distance between the OD and macula along the axis of symmetry is fixed at $5 r$, we define a region of interest (ROI) to be a wedge shaped region of this sector between $4 r$ and $5 r$ of the OD centre (shown in Fig.5, right).

2) Final Localization: Macula is a dark, roughly circular region and there are no vessels passing through it. Hence, pixel $p$ is the desired macula location if it satisfies the following conditions: 


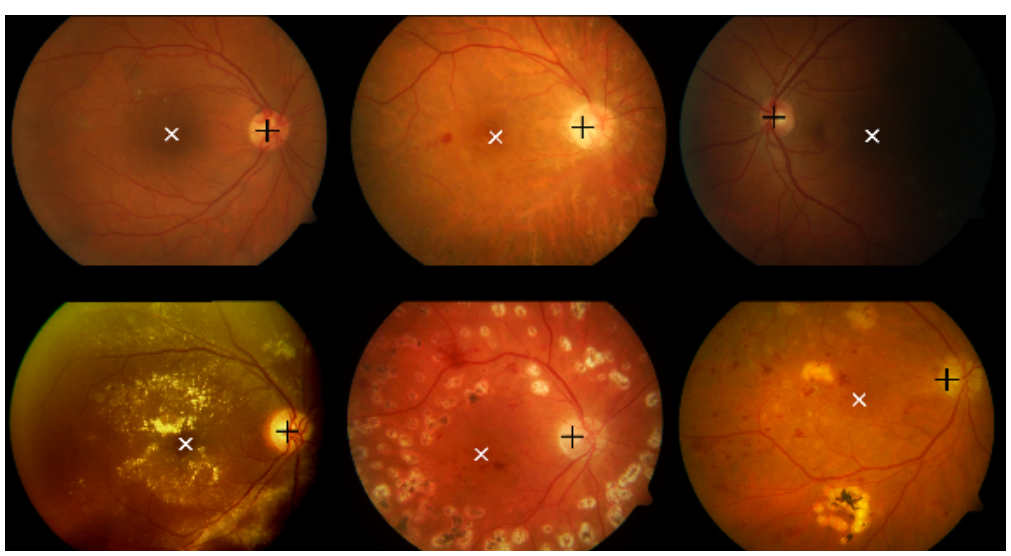

Fig. 6. Output of the algorithm on various images(OD is marked black and macula is marked white). Top row shows cases without lesions. Bottom row shows cases with lesions.

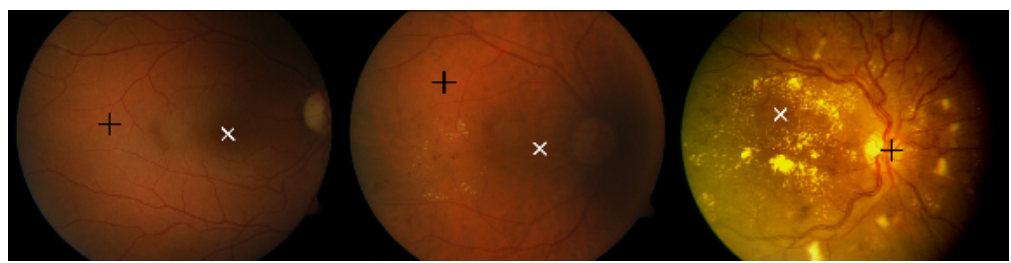

Fig. 7. Some images where the algorithm does not give correct output

TABLE II

COMPARISON OF DETECTION RATES FOR MACULA ON POPULAR DATASETS

\begin{tabular}{|c|c|c|}
\hline Dataset & $\begin{array}{c}\text { Detection Rate (\%) } \\
\text { Our Method }\end{array}$ & $\begin{array}{c}\text { Detection Rate (\%) } \\
{[17]}\end{array}$ \\
\hline Diaretdb0 (131 images) & 96.6 & 96.79 \\
\hline Diaretdb1 (89 images) & 96.9 & 98.74 \\
\hline Drive (20 images) & 90.0 & 91.73 \\
\hline Private Dataset (420 images) & 92.6 & - \\
\hline
\end{tabular}

TABLE III

COMPARISON OF DETECTION RATES FOR OD ON POPULAR DATASETS

\begin{tabular}{|c|c|c|}
\hline Dataset & $\begin{array}{c}\text { Detection Rate (\%) } \\
\text { Our Method }\end{array}$ & $\begin{array}{c}\text { Detection Rate (\%) } \\
{[17]}\end{array}$ \\
\hline Diaretdb0 (131 images) & 97.0 & 97.64 \\
\hline Diaretdb1 (89 images) & 96.2 & 97.79 \\
\hline Drive (20 images) & 100 & 100 \\
\hline Private Dataset (420 images) & 96.2 & - \\
\hline
\end{tabular}

1) The difference between the average brightness in the distant and immediate neighbourhoods of $p$ is large. This condition ensures that the region is locally dark.

2) The vessel density within the neighborhood of $p$ is small (ideally, it should be zero). This condition helps in distinguishing the macula from other dark lesions that may occur near vessels in the image.

3) The distance of $p$ from the nearest candidate line of symmetry is small. This is because the macula should ideally lie on one of these lines.

Each pixel $(\mathrm{x}, \mathrm{y})$ is represented in the search space by a 4dimensional vector $F_{i j}=\left\{S_{r}^{i j}, S_{1.5 r}^{i j}, V_{r}^{i j}, \rho_{L(m, c)}^{i j}\right\}$, where
$L(m, c)$ is the candidate line of symmetry closest to the pixel and the elements of this vector are as defined in table I.

We train a linear SVM classifier on the above 4-dimensional features to get a probability score for each pixel being the macula centre. The probability map is smoothed by a Gaussian filter and the pixel with maximum value is selected as the macula centre.

\section{EXPERIMENTS AND RESULTS}

We have evaluated our algorithm on 4 public datasets such as Diaretdb0[9], Diaretdb1[8] and Drive[16] and Messidor[1]. The SVM vectors were learned for each dataset by dividing it into training and testing sets in the ratio of 40:60. The results 
reported are the average results for each fold in the crossvalidation.

In fig 6, we show the qualitative results for OD and macula detection on a few sample retinal images. It can be seen that in the presence of variation in illumination and lesions our algorithm is able to detect OD and macula successfully. There are some failure cases of the proposed approach as shown in figure 7 . These are largely due to extreme instances of uneven illumination and the presence of very large-scale pathologies.

Quantitative evaluation was done using the same metric as used by[17]: detection is positive if it falls within the $\mathrm{OD} /$ macula regions in the ground truth. Detection rates for 3 public and one locally sourced (labelled as private) datasets are presented in table II and III. The results in [17] (shown in the last columns) are marginally higher than that obtained for our algorithm due to the employment of an ensemble of 5 different methods each, for macula and OD detection. Though the computational time is not reported in [17] it is expected to high due to the ensemble-based approach. In contrast, an unoptimized MATLAB implementation of our method on a 2 Ghz Core2 Duo machine takes around 20 seconds for the detection of both OD and macula. An optimized C++ implementation is expected to be even faster which is very attractive for screening applications.

Our algorithm achieved a detection rate of $98 \%$ for OD and 97\% macula on the Messidor[1] dataset with 1200 images.

\section{CONCLUSIONS}

In this work, we presented a bilateral symmetry based approach for the joint detection of OD and macula in a retinal image. Detection is achieved under a single algorithmic framework in which we take cue not just from local low-level features, but also from a global anatomical perspective where OD and macula exhibit a certain relationship with respect to the line of bilateral symmetry. The assessment results indicate that this approach is successful at handling challenges due to illumination changes and presence of lesions with the same level of performance as an ensemble-based approach based on 10 different algorithms. Since OD and macula detection form an important front end module in any CAD algorithm, a fast solution is critical for disease screening using CAD tools. The detection with the proposed method takes only 20 seconds at present. Further reduction is computation time and improvement is detection rate is possible by downsampling the images prior to detection as is done in [7]. The downsampling serves to reduce the noise.

Measures to improve the resizing need to be explored to achieve robustness to dataset variation. This can help in avoiding the need to train for each datasets as is done currently.

\section{ACKNOWLEDGEMENT}

This work was partially supported by the Department of Science and Technology, Government of India under DST/INT/NL/Biomed/P(3)/2011(G).

\section{REFERENCES}

[1] Kindly provided by the messidor program partners (see http://messidor.crihan.fr).

[2] AA-H Abdel-Razik Youssif, Atef $\mathrm{Z}$ Ghalwash, and AAS AbdelRahman Ghoneim. Optic disc detection from normalized digital fundus images by means of a vessels' direction matched filter. Medical Imaging, IEEE Transactions on, 27(1):11-18, 2008.

[3] Arturo Aquino, Manuel Emilio Gegúndez-Arias, and Diego Marín. Detecting the optic disc boundary in digital fundus images using morphological, edge detection, and feature extraction techniques. Medical Imaging, IEEE Transactions on, 29(11):1860-1869, 2010.

[4] Alan D Fleming, Sam Philip, Keith A Goatman, John A Olson, and Peter F Sharp. Automated assessment of diabetic retinal image quality based on clarity and field definition. Investigative ophthalmology \& visual science, 47(3):1120-1125, 2006.

[5] Marco Foracchia, Enrico Grisan, and Alfredo Ruggeri. Detection of optic disc in retinal images by means of a geometrical model of vessel structure. Medical Imaging, IEEE Transactions on, 23(10):1189-1195, 2004.

[6] Langis Gagnon, Marc Lalonde, Mario Beaulieu, and Marie-Carole Boucher. Procedure to detect anatomical structures in optical fundus images. Proc. SPIE Med. Image Process, 4322:1218-1225, 2001.

[7] A Giachetti, L Ballerini, E Trucco, and PJ Wilson. The use of radial symmetry to localize retinal landmarks. Computerized Medical Imaging and Graphics, 37(5):369-376, 2013.

[8] Tomi Kauppi, Valentina Kalesnykiene, Joni-Kristian Kamarainen, Lasse Lensu, Iiris Sorri, Asta Raninen, Raija Voutilainen, Hannu Uusitalo, Heikki Kälviäinen, and Juhani Pietilä. The diaretdb1 diabetic retinopathy database and evaluation protocol. In $B M V C$, pages 1-10, 2007.

[9] Tomi Kauppi, Valentina Kalesnykiene, Joni-Kristian Kamarainen, Lasse Lensu, Iiris Sorri, Hannu Uusitalo, Heikki Kälviäinen, and Juhani Pietilä. Diaretdb0: Evaluation database and methodology for diabetic retinopathy algorithms. Machine Vision and Pattern Recognition Research Group, Lappeenranta University of Technology, Finland, 2006.

[10] Marc Lalonde, Mario Beaulieu, and Langis Gagnon. Fast and robust optic disc detection using pyramidal decomposition and hausdorffbased template matching. Medical Imaging, IEEE Transactions on, 20(11):1193-1200, 2001.

[11] Huiqi Li and Opas Chutatape. Automatic location of optic disk in retinal images. In Image Processing, 2001. Proceedings. 2001 International Conference on, volume 2, pages 837-840. IEEE, 2001.

[12] Gareth Loy and Alexander Zelinsky. Fast radial symmetry for detecting points of interest. Pattern Analysis and Machine Intelligence, IEEE Transactions on, 25(8):959-973, 2003.

[13] Shijian Lu and Joo Hwee Lim. Automatic optic disc detection from retinal images by a line operator. Biomedical Engineering, IEEE Transactions on, 58(1):88-94, 2011.

[14] Meindert Niemeijer, Michael D Abràmoff, and Bram Van Ginneken. Segmentation of the optic disc, macula and vascular arch in fundus photographs. Medical Imaging, IEEE Transactions on, 26(1):116-127, 2007.

[15] Meindert Niemeijer, Michael D Abràmoff, and Bram van Ginneken. Fast detection of the optic disc and fovea in color fundus photographs. Medical image analysis, 13(6):859-870, 2009.

[16] Meindert Niemeijer, Joes Staal, Bram van Ginneken, Marco Loog, and Michael D Abramoff. Comparative study of retinal vessel segmentation methods on a new publicly available database. In Medical Imaging 2004 , pages 648-656. International Society for Optics and Photonics, 2004.

[17] Rashid Jalal Qureshi, Laszlo Kovacs, Balazs Harangi, Brigitta Nagy, Tunde Peto, and Andras Hajdu. Combining algorithms for automatic detection of optic disc and macula in fundus images. Computer Vision and Image Understanding, 116(1):138-145, 2012.

[18] Sribalamurugan Sekhar, Waleed Al-Nuaimy, and Asoke K Nandi. Automated localisation of retinal optic disk using hough transform. In Biomedical Imaging: From Nano to Macro, 2008. ISBI 2008. 5th IEEE International Symposium on, pages 1577-1580. IEEE, 2008.

[19] Chanjira Sinthanayothin, James F Boyce, Helen L Cook, and Thomas H Williamson. Automated localisation of the optic disc, fovea, and retinal blood vessels from digital colour fundus images. British Journal of Ophthalmology, 83(8):902-910, 1999.

[20] Thomas Walter and Jean-Claude Klein. Segmentation of color fundus images of the human retina: Detection of the optic disc and the vascular tree using morphological techniques. pages 282-287, 2001. 
[21] Frederic Zana and J-C Klein. Segmentation of vessel-like patterns using mathematical morphology and curvature evaluation. Image Processing, IEEE Transactions on, 10(7):1010-1019, 2001. 